\title{
Impaired emotion recognition is linked to alexithymia in heroin addicts
}

Giuseppe Craparo, Alessio Gori, Stefano Dell'Area, Giulia Costanzo, Silvia Fasciano, Antonia Tomasello, Carmelo M Vicario

Several investigations document altered emotion processing in opiate addiction.

Nevertheless, the origin of this phenomenon remains unclear. Here we examined the role of alexithymia in the ability (i.e. number of errors - accuracy - and reaction times - RTs) of thirty-one heroin addicts and thirty-one healthy controls to detect several affective expressions. Results show generally lower accuracy and higher RTs in the recognition of facial expressions of emotions for patients, compared to controls. The hierarchical multivariate regression analysis shows that alexithymia might be responsible of the between groups difference with respect to the RTs in emotion detection. Overall, we provide new insights in the clinical interpretation of affective deficits in heroin addicts suggesting a role of alexithymia in their ability to recognize emotions. 


\section{Impaired emotion recognition is linked to alexithymia in heroin}

\section{2 addicts}

4 Giuseppe Craparo 1, Alessio Gori 2, Stefano Dell'Aera 3, Giulia Costanzo 1, Silvia Fasciano 1,

5 Antonia Tomasello 1, Carmelo M. Vicario 4*

6

71 Faculty of Human and Social Sciences, Kore University of Enna, Italy

82 Department of Psychology, University of Florence, Italy

93 Department of Pathological Dependences, ASP - Enna, Italy

104 School of Psychology, Bangor University, Bangor, UK

*Correspondence to Carmelo M Vicario carmelo.vicario@bangor.ac.uk

15 Several investigations document altered emotion processing in opiate addiction. Nevertheless, 16 the origin of this phenomenon remains unclear. Here we examined the role of alexithymia in the 17 ability (i.e. number of errors - accuracy - and reaction times - RTs) of thirty-one heroin addicts 18 and thirty-one healthy controls to detect several affective expressions. Results show generally 19 lower accuracy and higher RTs in the recognition of facial expressions of emotions for patients, 20 compared to controls. The hierarchical multivariate regression analysis shows that alexithymia 21 might be responsible of the between groups difference with respect to the RTs in emotion 22 detection.

23 Overall, we provide new insights in the clinical interpretation of affective deficits in heroin 24 addicts suggesting a role of alexithymia in their ability to recognize emotions. 


\section{Introduction}

32 Drug addiction is a social-health problem with alarming statistics around the world.

33 From a social standpoint, the addiction to drugs is frequently associated with deficient

34 interpersonal relationships (Kornreich et al., 2002). This could be due to impaired processing of

35 emotional information related to social interactions (Kornreich, et al., 2003), though other factors

36 such as the degree of dependence and the drug related life style may be also be involved in such

37 impairments.

38 The study of emotional processing has represented a useful tool to explore interpersonal abilities

39 in addicts, given the role of emotion processing in social attitude (e.g., Niedenthal et al., 2005;

40 Vicario \& Newman, 2013; Vicario, Rafal \& Avenanti, 2015). Emotion recognition difficulties in

41 opiate addicts could affect interpersonal relationships, since the ability to accurately decode

42 facial expressions is an important component of functional/well-adjusted social interactions.

43 The current literature provides contrasting results about emotion recognition performance in this

44 clinical condition (see Kun \& Demetrovics, 2010 for a review). Kornreich et al. (2003) studied

45 emotion recognition in four different groups of participants: recently detoxified alcoholics (RA);

46 opiate addicts under methadone maintenance treatment (OM); detoxified opiate addicts (OA);

47 detoxified subjects with both alcohol and opiate dependence antecedents (DAO); Results showed

48 a lower emotion recognition accuracy in all clinical groups, compared to healthy controls. In

49 contrast, Martin et al., (2006) found that opiate users receiving methadone maintenance were 
50 more accurate than ex-opiate users in rehabilitation in recognizing facial expressions of disgust.

51 On the other hand, they were generally slower than controls in recognizing all expressions.

52 Finally, the study by Zhou et al. (2012) has shown that abstinent heroin abusers display a

53 heightened detection of negative emotion when searching stimulus displays with a varying

54 number of neutral faces for the positive or negative faces.

55 A psychological construct that could help to understand and disentangle these differences in 56 emotional recognition performance in opiate addicts is alexithymia. The term alexithymia was 57 coined by Sifneos (1973), to indicate "a deficit in the cognitive processing of emotions" (see

58 Taylor \& Bagby, 2013). Specifically, alexithymia is characterized by a reduced ability to identify and describe feelings, a difficulty to distinguish between different feelings, an externally oriented

60 cognitive approach to reality and a difficulty to modulate feelings (Porcelli et al., Taylor, 2014).

61 Alexithymia has been also associated with an impaired ability to recognize facially expressed

62 emotions (see Grynberg et al., 2012 for a recent review). For example, Prkachin, Casey and

63 PrKachin (2009) found an impaired ability in detecting affective expressions in populations with

64 alexithymia. In particular, the correlational analyses documented higher difficulty in recognizing

65 emotions such as sadness, anger, and fear. In similar fashion, Gil et al., (2009) reported a

66 significant and negative correlation between facial emotion recognition and alexithymia severity

67 in a group of twenty patients with somatoform disorders. The research by Lindsay and Ciarocchi

68 (2009) proposes 2 different potential explanations. One might refer to the mood, which is more

69 negative in substance abusers (Lindsay and Ciarocchi, 2009), compared to controls. In fact,

70 Haviland et al. (1994) found that negative mood (i.e., depression) predicts alexithymia. The

71 alternative explanation might refer to the inaccurate belief / low-motivation of addicts. As

72 explained by the authors, if people believe that they are not able to deal effectively with their 
73 emotions, they may be less motivated to do so. Less motivation, in turn, may lead to more

74 alexithymic behaviour. For these reasons, one could hypothesize a key role of alexithymia in the

75 emotional recognition deficit of addicts, given the relevance of this disorder in this clinical

76 condition (Craparo, 2014; Craparo et al., 2014a; Faraci et al., 2013; Torrado, Ouakinin \&

77 Bacelar-Nicolau, 2013; Craparo et al., 2014b; Craparo et al., 2014c). Indeed, as reported by

78 Farges et al., (2004), the prevalence of alexithymia in addicts is $43.5 \%$, compared to $24.6 \%$ in

79 healthy controls, as documented by using the TAS-20. The study of alexithymia in heroin

80 addiction is important, because it can provide insights about the origin of the emotional

81 processing deficit in this clinical population, as reported by the literature.

82 The research on addicts has also provided evidence of neurobiological alterations in addicts,

83 which might explain the emotional recognition deficit in this clinical population. For instance,

84 Kornreich et al. (2003) proposed that the origin of this deficit might be due to the chronic abuse

85 of drugs, which might cause deleterious effects on brain functions involved in decoding facial

86 expressions. This suggestion appears likely, given the evidence of the impaired activity of

87 several key regions for emotion (and reward) processing such as the insula, the cingulate cortex,

88 and the amygdale in addicts (Naqvi \& Bechara, 2009; Di chiara et al., 1999; Vicario et al., 2014).

89 However, these dysfunctions should not be conceived as separate from alexithymia, rather as

90 possible neural substrates. In fact, research has linked alexithymic features to abnormal activity

91 of the amygdale (Kugel et al., 2005) and the frontocingulate cortices (Berthoz et al., 2002).

92 In the current research we addressed, for the first time, the impact of alexithymia in the

93 emotional processing deficits of heroin addicts. Indeed, despite previous studies having shown

94 that these two phenomena are closely linked in other clinical populations such as adults with

95 somatoform disorders (Pedrosa et al., 2009), this remains to be investigated in heroin addicts. 
96 Thus, we measured participants' accuracy (i.e., proportion of correct answers) and reaction times

97 (RTs) in detecting affective expressions. According to previous studies documenting a role of

98 alexithymia in emotional recognition deficits, we expect to detect a positive relationship between

99 alexithymia severity and the difficulty in detecting negative emotions.

100

101 Methods

102 Participants

103 Sixty-two participants were selected for the current study. The drug addiction group was

104 composed of thirty-one participants (4 cocaine/heroin addicts, 25 males, average age $34.83 \pm$

105 8.6); The thirty-one healthy participants (control group) was composed by 25 males, average age

$10633.83 \pm 8.70)$. No between group difference has been detected with respect to the age $(\mathrm{t}=0.45$,

$107 \mathrm{p}=0.65)$. The four participants consuming both cocaine and heroin were excluded from the

108 analysis in order to have a homogeneous clinical group. The clinical group was selected in two

109 special sanitary treatment centers for drug addictions in Enna and Florence (Italy). All patients

110 were undergoing methadone treatment. The methadone dosage ranged between 20 a $80 \mathrm{mg}$ per

111 day. No psychosocial treatment was provided. Inclusion criteria were: i) diagnosis of heroin

112 addiction; ii) no previous experience of psychotherapy; iii) no diagnosis of severe mental illness

113 (e.g. psychosis, schizophrenia, major depression, anxiety, post-traumatic stress disorder); iv)

114 absence of other forms of addiction (according to the Addictive Behaviour Questionnaire, Caretti

115 in press); v) the use of other drugs. The clinical diagnosis was made by a psychiatrist, according

116 to the DSM V criteria, by structured clinical interview. Moreover, all patients underwent blood

117 and urine tests to confirm the type of drug metabolites. The urine test were negative for cannabis

118 use. The period of drug-taking ranged between 5 and 8 years. The healthy group was selected 
119 among university students and patients' relatives. They were screened through a clinical

120 interview, aiming to mainly exclude any form of addiction. This study was conducted in

121 accordance with the requirements of the Helsinki convention and approved by the local ethical

122 committee of Kore University. Informed consent was obtained from all participants.

123

124 Measures and Stimuli

125 The 20-item Toronto alexithymia scale (TAS-20) is a self-report scale used to measure

126 alexithymia. It is composed of three subscales: 1) Difficulty identifying feelings (DIF); 2)

127 Difficulty describing feelings (DDF); 3) Externally oriented thinking (EOT). Bagby et al. (1994)

128 proposed three cut-off scores in order to discriminate alexithymic $(\geq 61)$, borderline (score range

129 between 51 to 60$)$, and non alexithymic individuals $(\leq 50)$. A set of pictures were used,

130 representing the six basic emotions: happiness, sadness, fear, disgust, contempt and anger. For

131 our sample we used an Italian validated version made by Bressi et al. (1996). The scale was

132 administered, in a multicenter research project, in a nonclinical sample of 206 adults and a

133 nonclinical sample (medical and psychiatric disorders) of 642 subjects, showing good

134 psychometric properties (high reliability and validity; Bressi et al., 1996). In particular, as

135 reported by the authors, the goodness-of-fit was evaluated using four criteria recommended by

136 Cole (1987) and Marsh et al. (1988) viz., goodness-of-fit (GFI) $\geq 0.85$; adjusted goodness-of-fit

$137(\mathrm{AGFI}) \geq \mathrm{O} . \mathrm{BO}$; the root-mean-square residual $(\mathrm{RMSR}) \leq 0.10$; and the Tucker-Lewis index

138 (TLI) $\geq 0.80$. For the normal adult sample, the (GFI) (0.88), the (AGFI) (0.84), RMSR (0.07),

139 and TLI (0.80) all met the criteria standards, thus indicating adequacy of fit. Similar results were

140 obtained with the clinical out-patient sample, with the GFI (0.95), AGFI (0.93) RMSR (0.05),

141 and TLI (0.90) all meeting the criteria standards. 
142 We used photos of easy emotional intensity level from the Facial Action Coding System

143 (Ekman, Friesen \& Hager, 1978). Stimuli were presented in a random order via Personal

144 Computer.

145 Procedure

146 Participants were invited to fill out the TAS-20 questionnaire, evaluate and categorize facial

147 emotion expressions elicited from the photos representing the basic emotions (fear, anger,

148 disgust, happy, sadness, surprise, contempt). They were asked to name the emotion displayed in

149 each photo, presented in a random order and in absence of specific cues. Accuracy and reaction

150 times (RT) (using an electronic chronometer) were recorded. The administration of both

151 questionnaire and pictures was done in a silent room of the center for drug addicts, by using a

152 face-to-face method. The average time of each session was about 45 minutes.

153

154

155

156

157 severity.

164 For all tests, the level of statistical significance was set at $\mathrm{p}<0.05$. Data analyses were performed 165

\section{Data analysis}

We first used the two tailed t-test to compare the TAS-20 scores of our heroin addicts vs. control participants. Data including group and emotion as main factors were entered in a repeatedmeasures ANOVA to detect any between group difference with respect to the examined variables (Accuracy and RTs). Following this, we performed a hierarchical multivariate regression analysis using alexithymia (i.e., the TAS-20 score) as a predictor and emotional recognition performance (i.e., the overall average for both RTs and accuracy) as an outcome, controlling for the age of participants and the years of exposure to heroin. Finally, we performed a Pearson correlation analysis to investigate whether the exposure to heroin predicts alexithymia using the Statistica software, version 8.0, StatSoft, Inc., Tulsa USA and IBM SPSS Statistics 20. 


\section{Results}

168 Alexithymia index: As expected, we detected a significant between groups difference $(\mathrm{t}=4.36$, $169 \mathrm{p}<0.001)$ comparing TAS-20 scores of addicts $(\mathrm{M}=57.6 \pm 16.7 \mathrm{SD})$ with respect to controls

$170(\mathrm{M}=41.83 \pm 10.5 \mathrm{SD})$. This shows that control participants are, on the average, not affected by 171 alexithymia (i.e., TAS-20 score $\leq 50$ ), while addicts participants can be classified in the 172 borderline category with respect to the alexithymia index (i.e., TAS-20 score $>50$ and $<60$ ). No 173 gender difference is reported in both groups with respect to the TAS-20 scores $(\mathrm{p}>0.131)$.

174 Further analyses show significant difference between the three subscales, in both the clinical $175[\mathrm{~F}(2,52)=18.52, \mathrm{p}<0.001]$ and the control $[\mathrm{F}(2,52)=19.49, \mathrm{p}<0.001]$ samples. In particular, we 176 documented a lower score in the DIF sub-scale, compared to the DDF $(\mathrm{p}<0.001)$ and the EOT $177(\mathrm{p}<0.001)$ scales in the clinical sample; Moreover, we documented higher scores in the EOT sub178 scale, compared to the DIF $(\mathrm{p}<0.001)$ and DDF $(\mathrm{p}<0.001)$ subscales of the control sample.

179 Emotions recognition accuracy: The repeated measure ANOVA detected a significant main 180 effect for the Group factor $[\mathrm{F}(1,60)=5.68, \mathrm{p}=0.021]$, documenting a lower accuracy (i.e., 181 proportion of correct responses) for the clinical sample $(\mathrm{M}=0.650 \pm 0.037)$ in detecting

182 emotional stimuli compared to the control sample $(\mathrm{M}=0.774 \pm 0.035)$. We also detected a 183 significant main effect of the Emotion factor $[\mathrm{F}(6,354)=18.0, \mathrm{p}<0.001]$. However, the Group $\mathrm{x}$ 184 Emotion interaction term was not significant $[\mathrm{F}(6,354)=1.47, \mathrm{p}=0.189]$. No gender difference 185 was reported in emotion accuracy $(\mathrm{p}=0.851)$. The figure $1 \mathrm{~A}$ shows details concerning the 186 participants' performance with respect to the accuracy in the detection of the seven emotions.

187 Emotion recognition reaction times (RTS): We detected a significant main effect for the Group 188 factor $[\mathrm{F}(1,55)=4.85, \mathrm{p}=0.032]$, documenting higher $\mathrm{RTs}$ for the clinical sample $(\mathrm{M}=7.74 \pm$ 
189 0.608) in detecting emotional stimuli compared to the control sample $(\mathrm{M}=5.90 \pm 0.567)$. In

190 similar fashion, we documented a significant main effect for the Emotion factor $[F(6,156)=14.7$,

$191 \mathrm{p}<0.001]$. In contrast, no significant difference has been reported for the Group $\mathrm{x}$ Emotion

192 interaction term $[\mathrm{F}(6,156)=0.86, \mathrm{p}=0.522]$. The figure $1 \mathrm{~B}$ shows details concerning the

193 participants' performance with respect the RTs in the detection of the seven emotions. No gender

194 difference is reported in emotion RTs $(p=0.639)$
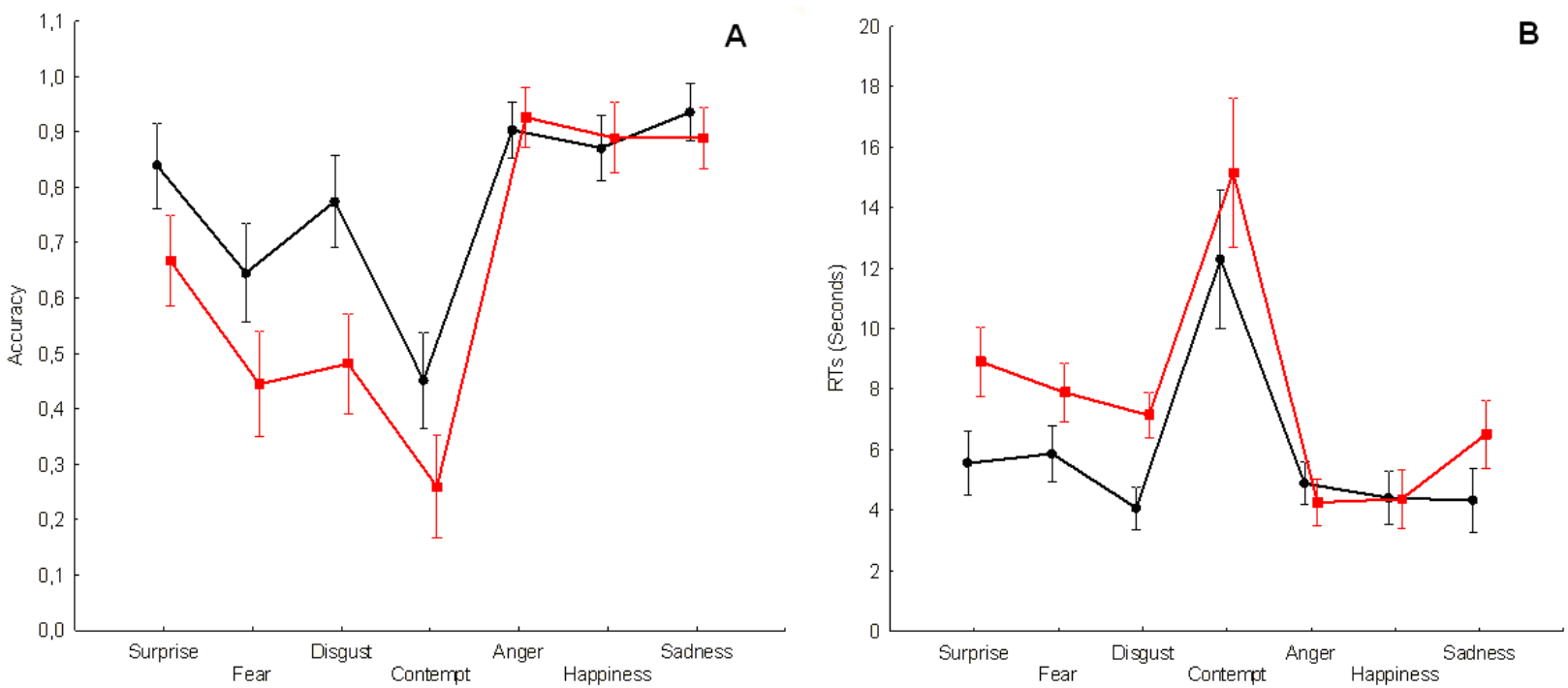

195

$\$$ Controls

196 Figure 1. $A$. The figure shows the proportion of correct responses (i.e. accuracy) for healthy

197 controls and heroin addicts (i.e. clinical sample) in the recognition of the facial expressions of emotions. The figure shows relevant differences for the recognition of Surprise, Fear, Disgust and Contempt. B. The figure shows RTs of healthy controls and heroin addicts (i.e. clinical sample) in the recognition of the facial expressions of emotions. The figure shows relevant differences only for the detection of Surprise, Fear, Disgust, Contempt and Sadness. Vertical Bars indicate standard error. 
203 Hierarchical multivariate regression analysis: With respect to the RTs variable, the initial

204 analysis (model 1, i.e. including the age and the years of exposure to heroin as predictors)

205 provides significant results $[\mathrm{F}(2,57)=4.97, \mathrm{p}=0.010]$, with no effect of age on performance

206 ( $\mathrm{p}=0.153)$, while the years of exposure to heroin was significant $(\mathrm{p}=0.036)$. This model explain

$20715 \%$ of the variance. The inclusion of the alexithymia (TAS-20 score) variable as predictor (i.e.

208 model 2, including age, years of exposure to heroin, alexithymia) cancels the effect of exposure

209 to heroin $(\mathrm{p}=0.420)$, while the effect of the alexithymia variable was significant $(\mathrm{p}=0.048)$. This

210 model explains $21 \%$ of the variance. With respect to accuracy parameters, both the model 1

$211[\mathrm{~F}(2,57)=2.68, \mathrm{p}=0.077]$ and the model $2[\mathrm{~F}(2,57)=2.40, \mathrm{p}=0.078]$ were not significant.

212

213 Discussion

214 Several studies (Craparo, 2014; Craparo et al., 2014a; Faraci et al., 2013; Torrado, Ouakinin \&

215 Bacelar-Nicolau, 2013; Craparo et al., 2014b; Craparo et al., 2014c) have linked alexithymia to

216 addiction. Moreover, alexithymia has been associated with deficits in emotion recognition

217 performance (e.g., see Taylor \& Bagby, 2013). However, the literature documents contrasting

218 results while examining emotion recognition performance in addicts, with evidence of both

219 lower (e.g., Kornreich et al., 2003) and higher (e.g., Martin et al., 2006; Zhou et al., 2012)

220 accuracy in this population. Nevertheless, no research has directly investigated the link between

221 alexithymia and emotion recognition performance in heroin addicts.

222 Overall, in the current research we show that heroin addicts are less accurate and slower in the

223 recognition of facial expressions of emotions, compared to healthy controls. This result

224 corroborates the research of Kornreich et al. (2003) which reported a similar pattern of results,

225 whilst appearing to contrast with the study of Zhou et al. (2012) who documented better 
226 performance in the recognition of negative emotions and also with the study of Martin et al.

227 (2006), who reporting higher accuracy in disgust recognition. However, our data corroborates the

228 results of Zhou et al. (2012), documenting slower RTs of heroin addicts in the overall emotion

229 recognition, although only for the recognition of the expressions of negative emotions.

230 A novel result emerging from the hierarchical multivariate regression analysis, which fits with

231 our main research hypothesis, is that alexithymia explains the between groups difference with

232 respect to the RTs. This suggests that the poor performance of heroin addicts documented in our

233 study can be explained, at least in part, by referring to their alexithymia traits. This is also in

234 agreement with the research on somatoform disorder patients (Pedrosa Gil et al., 2009) and on

235 Asperger syndrome (Kätsyri et al., 2008). Finally, we detected a positive correlation trend

236 between years of exposure to heroin and TAS-20 scores, suggesting that the progressive use of

237 this drug might increase alexithymia severity.

238 The slower performance in emotion recognition, as reported in our work, which depends on 239 alexithymia, might originate from the effect of heroin exposure to the neural circuits that appear

240 to be critical in emotion processing, such as the insula, the amygdala, orbitofrontal cortex, the 241 anterior cingulated cortex and the basal ganglia (e.g., see Adolphs, 2002 for a review). In fact,

242 there is evidence (Liu, et al., 2011; Li et al., 2003) of functional dysregulation of these regions in

243 heroin abusers. Interestingly, these neural regions have been reported to be impaired in people

244 with alexithymia (Berthoz et al., 2002; Mantani et al., 2005). Therefore, according to our

245 correlation analysis, one could hypothesize that the long term exposure to heroin might at least

246 exacerbate alexithymia, resulting in this effect on the neural system.

247 Alexithymia has been well documented across different disorders, including autism and eating 248 disorders. This may explain, at least in part, the emotional difficulties across these populations. 
249 This leads to speculate that alexithymia intervention programs may lead to improvements in

250 social and emotional abilities across a wide range of clinical conditions.

251 Future works investigating emotion processing in addiction might expand the current

252 investigation by exploring the links between alexithymia, psychopathic traits, withdrawal 253 symptoms and emotion recognition.

254

255 Limitations

256 Several limitations should be mentioned with respect to the current study. Firstly, the distribution

257 of alexithymia scores and the gender variable were not balanced between our two groups of 258 participants. The clinical sample has been reduced because four participants were consuming 259 both cocaine and heroin. Further limitations might be referred to the absence of information 260 about the years of scholastic education; the methadone dosage and the presence of psychopathic 261 traits. In similar fashion, we did not investigate psychopathological symptoms in relation to 262 alexithymia. Finally, RTs were detected by using a stopwatch rather than a computerized system.

\section{References}

265 Adolphs R. 2002. Neural systems for recognizing emotion. Current Opinion in Neurobiology 12: 266 169-77 Review.

267 Bagby RM, Taylor GJ Parker JDA. 1994. The twenty-item Toronto Alexithymia Scale. II. 268 Convergent, discriminant, and concurrent validity. Journal of Psychiatric Research 38: 33-40.

269 Berthoz S, Artiges E, Van De Moortele PF, Poline JB, Rouquette S, Consoli SM, Martinot JL 270 2002. Effect of impaired recognition and expression of emotions on frontocingulate cortices: an 271 fMRI study of men with alexithymia. American Journal of Psychiatry 159: 961-7. 
272 Blair RJ, Coles M. 2000. Expression recognition and behavioural problems in early adolescence.

273 Cognitive Development 15: 421-434.

274 Blair RJ, Morris JS, Frith CD, Perrett DI, Dolan RJ 1999. Dissociable neural responses to facial 275 expressions of sadness and anger. Brain 122: 883-893

276 Bressi, C., Taylor, G. J, Parker, J. D. A., Bressi, S., Brambilla, V., Aguglia, E., Allegranti, S., 277 Bongiorno, A., Giberti, F., Bucca, M., Todarello, O., Callegari, C., Vender, S., Gala, C., 278 Invernizzi, G. (1996), "Cross validation of the factor structure of the 20-Item Toronto 279 Alexithymia Scale: an Italian multicenter study", Journal of Psychosomatic Research, 41, pp. $280 \quad 551-559$.

281 Caretti V. In press. Addictive Behav Questionn (ABQ). Hogrefe. firenze.

282 Craparo G. 2014. The role of dissociation, affect dysregulation, and developmental trauma in 283 sexual addiction. Clinical Neuropsychiatry 11: 86-90.

284 Craparo G, Ardino V, Gori A, Caretti V. 2014a. The Relationships between Early Trauma, 285 Dissociation, and Alexithymia in Alcohol Addiction. Psychiatric Investigation 11: 330-335.

286 Craparo G, Gori A, Mazzola E, Petruccelli I, Pellerone M, Rotondo G. 2014b. Posttraumatic 287 stress symptoms, dissociation, and alexithymia in an Italian sample of flood victims. 288 Neuropsychiatric Diseases Treatment 10: 2281-2284. doi: 10.2147/NDT.S74317

289 Craparo G, Gori A, Petruccelli I, Cannella V, Simonelli, C. 2014. Intimate Partner Violence: 290 Relationships Between Alexithymia, Depression, Attachment Styles, and Coping Strategies of 291 Battered Women. Journal of Sexual Medicine 11: 1484-1494. doi: 10.1111/jsm.12505. Epub 2922014 Mar 13

293 De Bernardis D, D'Albenzio A, Gambi F, Sepede G, Valchera A, Conti CM, Fulcheri M, Cavuto 294 M, Ortolani C, Salerno RM, Serroni N, Ferro FM. 2009. Alexithymia and Its Relationships with 
295 Dissociative Experiences and Internet Addiction in a Nonclinical Sample. Cyber Psychological 296 Behavior 12: 67-69.

297 Di Chiara G, Tanda G, Bassareo V, Pontieri F, Acquas E, Fenu S, Cadoni C, Carboni E. 1999. 298 Drug addiction as a disorder of associative learning. Role of nucleus accumbens shell/extended 299 amygdala dopamine. Annals of the New York Academic Science 877: 461-485.

300 Ekman P, Friesen W, Hager JC 1978. Facial Action Coding System Investigator's Guide. 1978 301 Palo Alto: Consulting Psychol Press.

302 Faraci P, Craparo G, Messina R, Severino S. 2013. Internet Addiction Test (IAT): which is the 303 best factorial solution? Journal of Medical Internet Research 15: e225. doi: 10.2196/jmir.2935.

304 Farges F, Corcos M, Speranza M, Loas G, Perez-Diaz F, Venisse JL Jeammet, P. 2014. 305 Alexithymia, depression and drug addiction. Encephale 30: 201-11.

306 Gil FP, Ridout N, Kessler H, Neuffer M, Schoechlin C, Traue HC, Nickel M. 2006. Facial

307 Emotion Recognition and Alexithymia in Adults with Somatoform Disorders. Depression and 308 Anxiety 26: E26-E33.

309 Goldin PR, Hutcherson CA, Ochsner KN, Glover GH, Gabrieli JD, Gross JJ 2005. The neural

310 bases of amusement and sadness: A comparison of block contrast and subject-specific emotion

311 intensity regression approaches. Neuroimage 27: 26-36.

312 Gori A, Craparo G, Sareri GI, Caretti V, Giannini M, Meringolo P. 2014. Antisocial and 313 psychopathic personalities in a sample of addicted subjects: Differences in psychological 314 resourced, symptoms, alexithymia and impulsivity. Comprehensive Psychiatry 55: 1580-1586. 315 doi: 10.1016/j.comppsych.2014.05.023.

316 Grynberg D, Chang B, Corneille O, Maurage P, Vermeulen N, Berthoz S, Luminet O. (2012).

317 Alexithymia and the processing of emotional facial expressions (EFEs): systematic review, 
318 unanswered questions and further perspectives. PLoS One 7: e42429. doi:

319 10.1371/journal.pone.0042429.

320 Haviland MG, Hendryx MS, Shaw DG, Henry JP. 1994. Alexithymia in women and men

321 hospitalized for psychoactive substance dependence. Comprehensive Psychiatry 35:124-128

322 Kätsyri J, Saalasti S, Tiippana K, von Wendt L, Sams M (2008) Impaired recognition of facial

323 emotions from low-spatial frequencies in Asperger syndrome. Neuropsychologia 46: 1888-1897.

324 doi: 10.1016/j.neuropsychologia.2008.01.005

325 Kornreich C, Philippot P, Foisy ML, Blairy S, Raynaud E, Dan B, Hess U, Noël X, Pelc I,

326 Verbanck P. 2002. Impaired emotional facial expression recognition is associated with

327 interpersonal problems in alcoholism. Alcohol Alcohol 37: 394-400.

328 Kornreich C, Foisy ML, Philippot P, Dan B, Tecco J, Noël X, Hess U, Pelc I, Verbanck P. 2003.

329 Impaired emotional facial expression recognition in alcoholics, opiate dependence subjects,

330 methadone maintained subjects and mixed alcohol-opiate antecedents subjects compared with

331 normal controls. Psychiatry Research 3: 251-260.

332 Kugel H, Eichmann M, Dannlowski U, Ohrmann P, Bauer J, Arolt V, Heindel W, Suslow T.

333 2008. Alexithymic features and automatic amygdala reactivity to facial emotion. Neuroscience

334 Letters 435: 40-4.

335 Kun B, Demetrovics Z. 2010. Emotional intelligence and addictions: a systematic review.

336 Substance Use \& Misuse 45: 1131-1160

337 Lander GC, Lutz-Zois CJ, Rye MS, Goodnight JA. 2012. The differential association between

338 alexithymia and primary versus secondary psychopathy. Personality and Individual Differences, $33952: 45-50$ 
340 Lindsay, J., Ciarrochi, J. Substance abusers report being more alexithymic than others but do not

341 show emotional processing deficits on a performance measure of alexithymia Addiction

342 Research \& Theory, 17: 315-321

343 Li Q, Wang Y, Zhang Y, Li W, Zhu J, Zheng Y, Chen J, Zhao L, Zhou Z, Liu Y, Wang W, Tian

344 J 2013. Assessing cue-induced brain response as a function of abstinence duration in heroin-

345 dependent individuals: an event-related fMRI study. PLoS One 8: e62911. doi:

346 10.1371/journal.pone.0062911.

347 Liu J, Qin W, Yuan K, Li J, Wang W, Li Q, Wang Y, Sun J, von Deneen KM, Liu Y, Tian J.

3482011 Interaction between dysfunctional connectivity at rest and heroin cues induced brain

349 responses in male abstinent heroin-dependent individuals. PLoS One 6: e23098. doi:

350 10.1371/journal.pone.0023098.

351 Mantani T, Okamoto Y, Shirao N, Okada G, Yamawaki S 2005. Reduced activation of posterior

352 cingulate cortex during imagery in subjects with high degrees of alexithymia: a functional

353 magnetic resonance imaging study. Biological Psychiatry, 57: 982-990.

354 10.1016/j.biopsych.2005.01.047.

355 Martin L, Clair J, Davis P, O'Ryan D, Hoshi R, Curran HV. 2006. Enhanced recognition of facial

356 expressions of disgust in opiate users receiving maintenance treatment. Addiction 101: 15983571605.

358 Naqvi NH, Bechara A. 2009. The hidden island of addiction: the insula. Trends in Neuroscience 359 1: 56-67.

360 Niedenthal PM, Barsalou LW, Winkielman P, Krauth-Gruber S, Ric F. 2005. Embodiment in 361 attitudes, social perception, and emotion. Personality Social Psychological Review 3: 184-211. 
362 Pedrosa Gil F, Ridout N, Kessler H, Neuffer M, Schoechlin C, Traue HC, Nickel M. 2009. Facial

363 emotion recognition and alexithymia in adults with somatoform disorders. Depression and

364 Anxiety 26:E26-33.

365 Porcelli P, Affatati V, Bellomo A, De Carne M, Todarello O, Taylor GJ. 2004. Alexithymia and

366 psychopathology in patients with psychiatric and functional gastrointestinal disorders.

367 Psychoterapy and Psychosomatic 73: 84-91.

368 PrKachin GC, Casey C, PrKachin KM. 2009. Alexithymia and perception of facial expressions

369 of emotion. Personality and Individual Differences 46: 412-417.

370 Rybakowski J, Ziółkowski M, Zasadzka $\quad$ T, $\quad$ Brzeziński $\quad$ R. 1998.

371 High prevalence of alexithymia in male patients with alcohol dependence. Drug and

372 Alcohol Dependence 21: 133-6.

373 Savov S, Atanassov N 2013. Deficits of affect mentalization in patients with drug addiction:

374 theoretical and clinical aspects. ISRN Addict. :250751. doi: 10.1155/2013/250751.

375 Sifneos PE. 1973. The prevalence of 'alexithymic' characteristics in psychosomatic patients.

376 Psychotherapy and Psychosomatic 22: 255-262.

377 Stevens D, Charman T, Blair RJ 2001. Recognition of emotion in facial expressions and vocal

378 tones in children with psychopathic tendencies. Journal of General Psychology 162: 201-211.

379 Taylor GJ, Bagby RM. 2013. Psychoanalysis and Empirical Research: The Example of

380 Alexithymia. Journal of the American Psychiatric Association 61: 99-133. doi:

$381 \quad 10.1177 / 0003065112474066$

382 Taylor, G., Bagby, R. and Parker, J. 1997. The development and regulation of affects.

383 in Disorders of Affect Regulation: Alexithymia in Medical and Psychiatric Illness, G. Taylor, R.

384 Bagby, and J. Parker, Eds., Cambridge University Press, Cambridge, UK. 
385 Torrado MV, Ouakinin SS, Bacelar-Nicolau L. 2013. Alexithymia, Emotional Awareness and

386 Perceived Dysfunctional Parental Behaviors in Heroin Dependents. International Journal of

387 Mental Health Addiction 11: 6.

388 Vassileva J, Petkova P, Georgiev S, Martin EM, Tersiyski R, Raycheva M, Velinov V, Marinov

389 P. 2007. Impaired decision-making in psychopathic heroin addicts. Drug and Alcohol

390 Dependence 86:287-289

391 Vicario, C.M., Rafal, R.D., Avenanti, A. (2015). Counterfactual thinking affects the excitability

392 of the primary motor cortex. Cortex. $65 \mathrm{C}: 139-148$

393 Vicario CM, Komeilipoor N, Cesari P, Rafal RD, Nitsche MA. 2014. Enhanced corticobulbar

394 excitability in chronic smokers during visual exposure to cigarette smoking cues. The Journal of

395 Psychiatry and Neuroscience 39: 232-8.

396 Vicario CM, Newman A 2013. Emotions affect the recognition of hand gestures. Frontiers in

397 Human Neuroscience 7, 906. doi: 10.3389/fnhum.2013.00906

398 Zhou Y, Zhu H, Jin X, Li X, Zhang M, Zhang F, Shen $\quad$ M. 2012.

399 Biased attention towards negative schematic expression in abstinent heroin abusers. Journal of

400 Behavioral Theraphy and Experimental Psychiatry 43: 705-10. doi: 10.1016/j.jbtep.2011.1 